\title{
Influence of Oscillating Flow on LDL Transport and Wall Shear Stress in the Normal Aortic Arch
}

\author{
J. Soulis ${ }^{1, *}$, G. Giannoglou ${ }^{2}$, M. Dimitrakopoulou ${ }^{1}$, V. Papaioannou ${ }^{1}$, S. Logothetides ${ }^{3}$ and \\ D. Mikhailidis ${ }^{4}$
}

${ }^{1}$ Fluid Mechanics, School of Engineering, Demokrition University of Thrace, Xanthi, Greece

${ }^{2}$ AHEPA University General Hospital, $1^{\text {st }}$ Cardiology Department, Aristotle University of Thessaloniki, Thessaloniki, Greece

${ }^{3}$ AHEPA Physics Department, Aristotle University of Thessaloniki, Thessaloniki, Greece

${ }^{4}$ Dept. of Clinical Biochemistry (Vascular Prevention Clinics) and Dept. of Surgery, Royal Free Hospital campus, University College Medical School, University College London, London, UK

\begin{abstract}
Lipid accumulation in the aortic wall is an important factor in the development of atherosclerosis. The Low Density Lipoprotein (LDL) at the surface of the endothelium in relation to Wall Shear Stress (WSS) in the normal human aortic arch under unsteady, normal flow and mass conditions was computationally analysed. Concave sides of the aortic arch exhibit, relatively to the convex ones, elevated LDL levels at the surface of the endothelium for all time steps. At the peak systolic velocity, the LDL level reaches a value $23.0 \%$ higher than that at entrance in the ascending-descending aorta region. The corresponding LDL levels at the surface of the endothelium for the near minimum entrance velocity instant reaches $26.0 \%$. During the cardiac cycle, the highest area averaged normalized LDL taken up as compared to the lowest one is $0.69 \%$. WSS plays an important role in the lipid accumulation. Low WSS regions are exposed to high LDL levels at the surface of the endothelium. Regions of elevated LDL levels do not necessarily co-locate to the sites of lowest WSS. The near wall paths of the velocities might be the most important factor for the elevated LDL levels at the surface of the endothelium.
\end{abstract}

Keywords: Unsteady Low-density lipoprotein Transport, Wall Shear Stress, Atherosclerosis, Aortic Arch.

\section{INTRODUCTION}

Lipid accumulation in the aortic wall is an important factor in the development of atherosclerosis. Disturbed blood flow and occurrence of atherosclerotic plaques at certain sites of human arteries are strongly correlated [1]. The flow of blood and the transport of macromolecules in the vascular system [2] are essential to understand atherogenesis [3]. Biomechanical factors, such as Wall Shear Stress (WSS), blood viscosity and flow velocity, may be responsible for the localization and progression of atherosclerosis $[4,5]$. Besides the spatial distribution of flow parameters, their temporal variation during the cardiac cycle has been proposed as an atherogenic factor [6]. Complex configuration of the vessel promotes flow disturbances with low time-averaged WSS and high shear stress temporal oscillations during the cardiac cycle [6]. The application of unsteady flow constitutes a challenging issue, since it demands computational power and analysis time. The WSS may affect endothelial permeability [7]. Regional variations in the permeability of arterial endothelium may contribute to the localization of atheroscle-

*Address correspondence to this author at the Fluid Mechanics, School of Engineering, Demokrition University of Thrace, 67100 Xanthi, Greece; Tel: ++302541079617; Fax: ++302310994838; E-mail: jvsoulis@med.auth.gr rosis $[8,9]$. Luminal surface level of Low Density Lipoprotein (LDL) transport across the artery wall is considered to be important in atherogenesis $[10,11]$. Elevated LDL colocalize with known sites of atherosclerotic plaque development, [2]. Atherosclerotic regions are also low WSS regions and consequently it was difficult to determine whether it was low WSS, abnormal mass transfer or both that were contributing to atherosclerosis [2]. Oscillating flow on LDL transport in the arterial wall showed that the steady flow assumption is inadequate and the instantaneous hemodynamic conditions have important influence on LDL transmural transport in arteries with disturbed and complicated flow patterns [12]. The accumulation of LDL in vascular areas featuring a highly disturbed flow was examined, [13]. Geometrical parameters such as curvature and variations of the luminal section strongly influence the LDL within the wall. Realizing that the research produced in macromolecular transport in curved 3D blood vessels was rare, steady and unsteady flows and mass simulation was analyzed [14]. The effect of near-wall blood flow velocity and plasma filtration velocity across the arterial wall on luminal surface concentration of LDL and the uptake of tritium-cholesterol were investigated [15]. These results indicate that lipids accumulate at the luminal surface in areas where blood flow velocity and WSS are low and where the permeability of the endothelial layer is enhanced. 


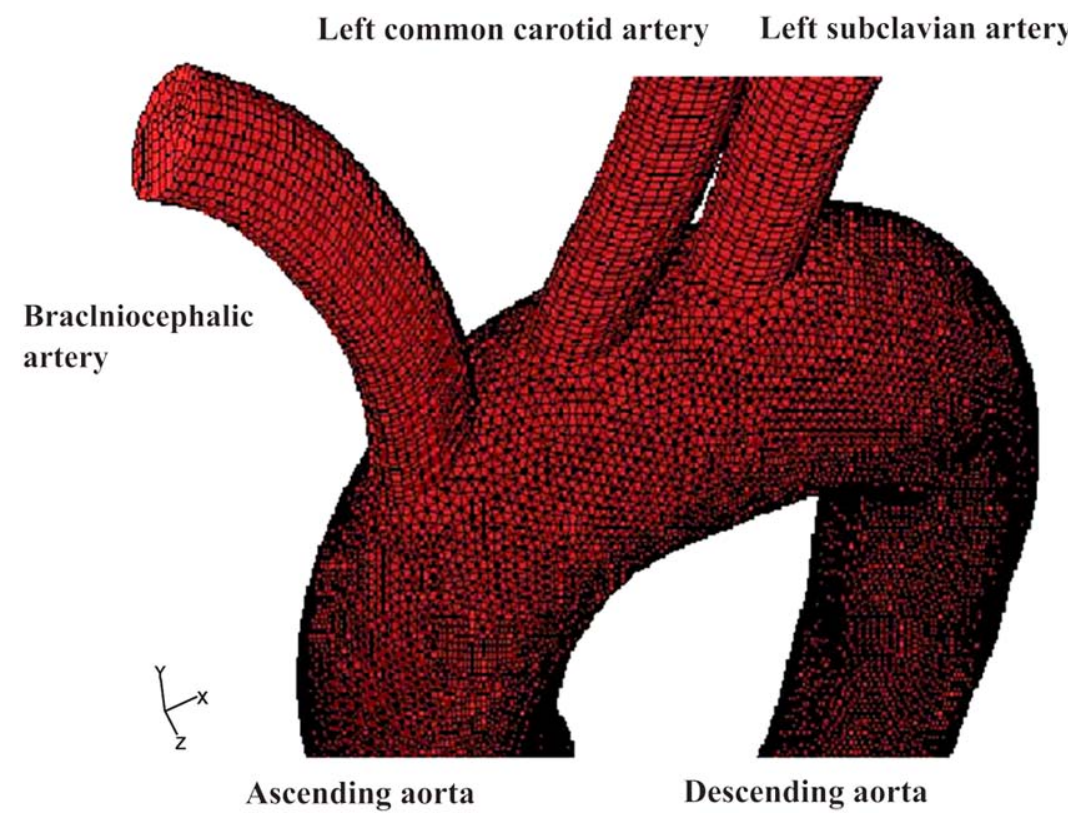

Fig. (1). Aortic arch geometry and non-structured computational grid including the: ascending aorta, descending aorta, brachiocephalic artery, left common carotid artery and left subclavian artery.

There have been very few studies investigating the fluidwall coupled mass transport under oscillating flow conditions $[12,14,16]$. The current work analyzes the influence of oscillating flow and LDL mass transport patterns over the normal human aortic arch. The purpose is to elucidate the association of low WSS-elevated LDL levels at the surface of the endothelium in relation to atherosclerotic plaque localization over the physiological human aortic arch during the normal cardiac pulse. Emphasis is put into a) LDL, b) WSS, c) differentiation of the LDL and WSS between concave (outer) and convex (inner) aortic arch sides as well as into d) temporal differentiation of the LDL between end systolic and end diastolic periods. We demonstrate that due to the semi-permeable nature of the arterial walls, high LDL levels at the surface of the endothelium occur at certain regions of the normal aortic arch.

\section{MATERIALS AND METHODOLOGY}

The 3D geometry of the normal aortic arch computational model was generated using data compiled from several sources, Fig. (1). The computational model includes the ascending aorta, descending aorta, brachiocephalic artery, left common carotid artery and left subclavian artery.

The assumptions made about the nature of the flow are that it is 3D, unsteady, laminar, isothermal, with no external forces applied on it while the aortic arch wall is comprised from non-elastic and semi-permeable material. The blood is considered to be non-Newtonian fluid obeying the power law. The flow equation is coupled with the convectiondiffusion equation. The diffusion flux of LDL arises due to concentration gradients. In the absence of systematic and reliable experimental data for human arteries, we assume the molecular diffusivity $D$ to be constant and equal to $15.0 \times 10^{-12} \mathrm{~m}^{2} / \mathrm{s}[9,17]$. The infiltration velocity $V_{w}$ is set equal to $0.6 \times 10^{-8} \mathrm{~m} / \mathrm{s}$ [18]. There is a limited amount of experimental data regarding the endothelial permeability $K$ or else mass transfer coefficient. Specifications of $K$ values are sometimes unreliable. For current analysis purposes this value is set equal to $2.0 \times 10^{-10} \mathrm{~m} / \mathrm{s}$, [9].

A typical aortic arch blood flow waveform, shown in Fig. (2), is applied over the entire entrance cross-section. The waveform mimics typical aortic blood averaged flow velocity under resting condition. From the entire velocity waveform, lasting $800.0 \mathrm{msec}$, two characteristic time-points are selected for detail analysis. The first instant refers to peak systole occurring $123 \mathrm{msec}$ after the systole onset with 0.32 $\mathrm{m} / \mathrm{s}$ entrance velocity. The second instant refers to near minimum entrance velocity of $0.005 \mathrm{~m} / \mathrm{s}$ corresponding to $522.75 \mathrm{msec}$. The inlet based Reynolds numbers range from 3.5 (minimum) to 475 (peak).

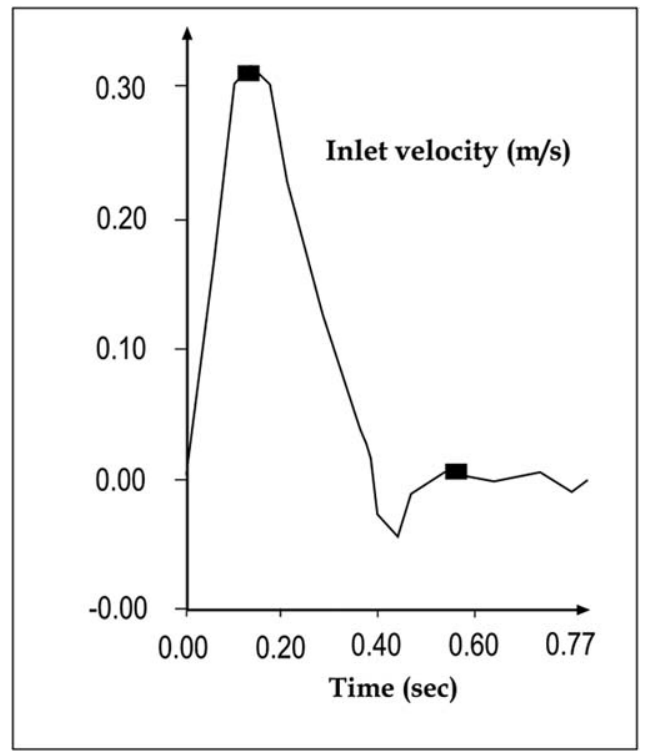

Fig. (2). Average physiological human aortic arch velocity waveform. Computational analysis results refer to inlet flow velocities of a) $0.32 \mathrm{~m} / \mathrm{s}$ (peak systole) and b) $0.005 \mathrm{~m} / \mathrm{s}$ (near minimum entrance velocity). 
A uniform constant concentration $C_{o}$ of LDL is applied at the orifice of the ascending aorta. At the descending aorta, brachiocephalic artery, left common carotid artery and left subclavian artery outlets, the gradient of LDL concentration along the vessels is set equal to zero (zero flux, Newmann condition). Flow discharges are set analogous to the third power of the branching vessel inlet diameter according to Murray's law [19].

The applied endothelium boundary condition at the semipermeable aortic walls states that the net amount of LDL per unit area passing from it to the vessel wall is determined by the difference of the mass flow carried to the vessel wall by infiltration flow and the amount of flow which diffuses back to the main vessel flow. It is known that at arterial walls the LDL concentration values are higher than those of the vessel bulk flow. Furthermore, LDL concentration increases with increasing infiltration velocity.

\section{RESULTS}

The calculated LDL levels at the surface of the endothelium $C w$ are normalized with the inlet value of $C o \quad(=1.3$ $\mathrm{mg} / \mathrm{ml}$ ). WSS contours show its magnitude and not its spatial direction.

\subsection{WSS in the Ascending-Descending Aorta}

WSS $\left(\mathrm{N} / \mathrm{m}^{2}\right)$ magnitude contour plots for the ascendingdescending aorta of the aortic arch for the selected time instants are shown in Figs. $(\mathbf{3 a}, \mathbf{b})$. The convex side of the aortic arch exhibits, relatively to the concave side, high WSS values. High WSS is encountered at the convex side of the

a)
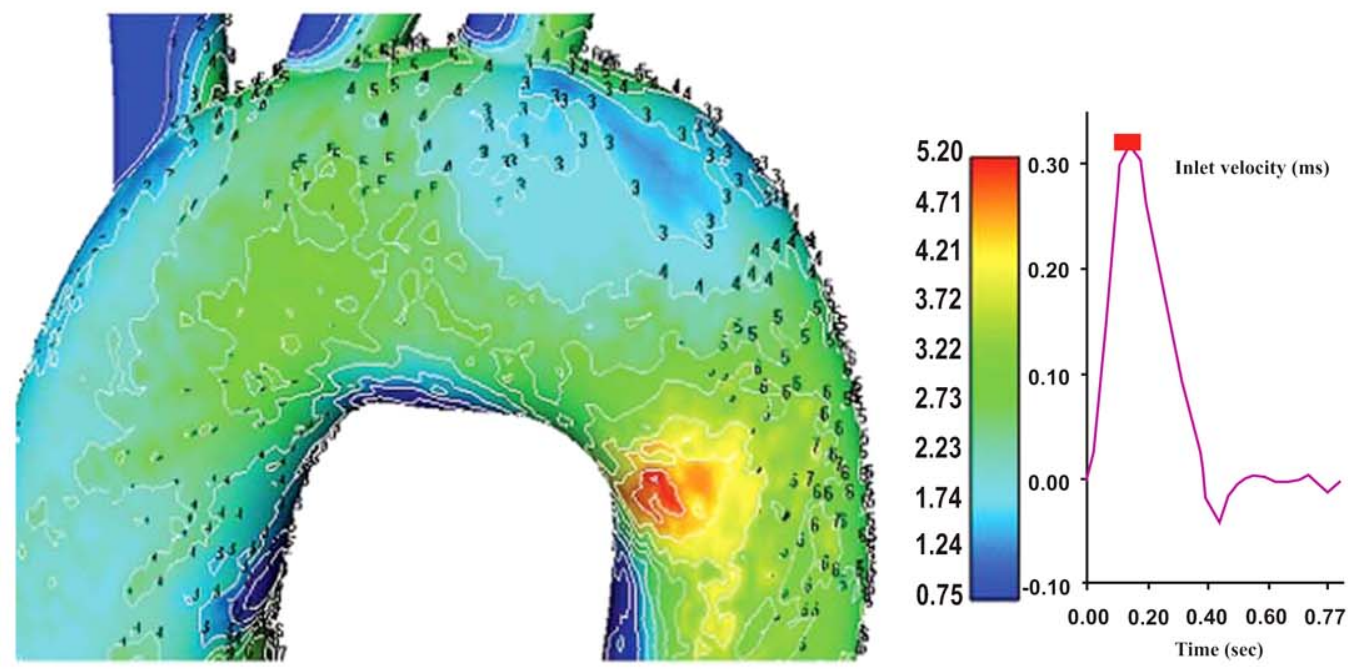

b)
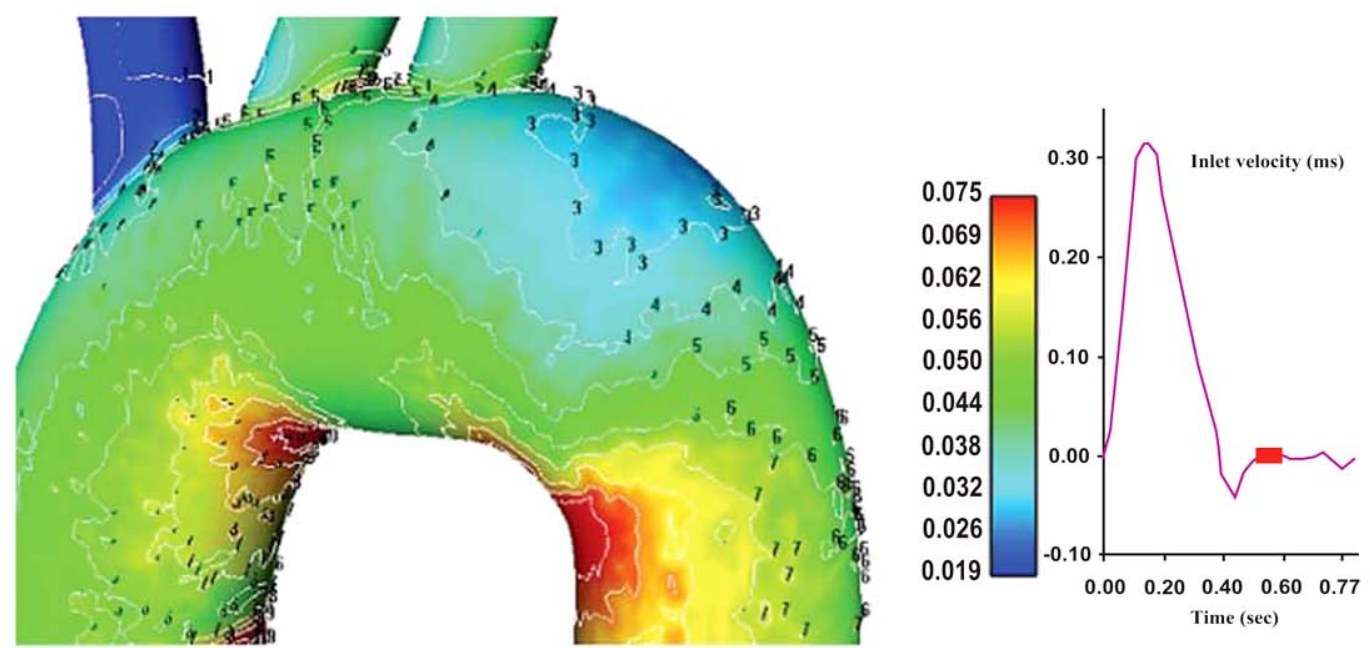

Fig. (3). Colour-coded contour plots of the Wall Shear Stress $\left(\mathrm{N} / \mathrm{m}^{2}\right)$ magnitude for the ascending-descending aorta at a) peak systole and b) near minimum entrance velocity, respectively. 
end ascending and early descending aorta at all pulse wave times. Particular low WSS values appear along the concave parts of the ascending-descending aorta at regions located just downstream to the left subclavian artery.

\subsection{LDL in the Ascending-Descending Aorta}

Contour plots of the normalized luminal surface concentration $\mathrm{Cw} / \mathrm{Co}$ for the ascending-descending aorta, at the selected characteristic time instants, are shown in Figs. (4a, b). At all time instants, the concave side of the aortic arch exhibits high LDL levels compared with the convex side. Low LDL levels at the surface of the endothelium are encountered at the end ascending and the early descending convex side of the aorta, while the main convex side encounters relatively high LDL levels Figs. (5a, b). At peak systolic pressure, the lowest $\mathrm{Cw} / \mathrm{Co}$ value is 1.13. High LDL values appear on the luminal surface of the wall along the concave parts of the ascending-descending aorta. Regions with elevated LDL levels at the surface of the endothelium occur at the concave ascending-descending aortic arch just downstream to the left subclavian artery.

\subsection{LDL in the Brachiocephalic Artery, Left Common Carotid Artery and Left Subclavian Artery}

$\mathrm{Cw} / \mathrm{Co}$ contour plots for the brachiocephalic artery, left common carotid artery and left subclavian artery at the specific time instants are shown in Figs. $(\mathbf{6 a}, \mathbf{b})$. Elevated LDL levels at the surface of the endothelium are observed in all vessels. This is due to low flow velocities which in turn gives rise to low WSS. The particular LDL levels are strongly affected by vessel curvature. As a rule, concave parts exhibit high luminal surface LDL. However, depending upon the particular bend side and the incoming flow velocity distribution, convex parts can exhibit high LDL levels.

a)
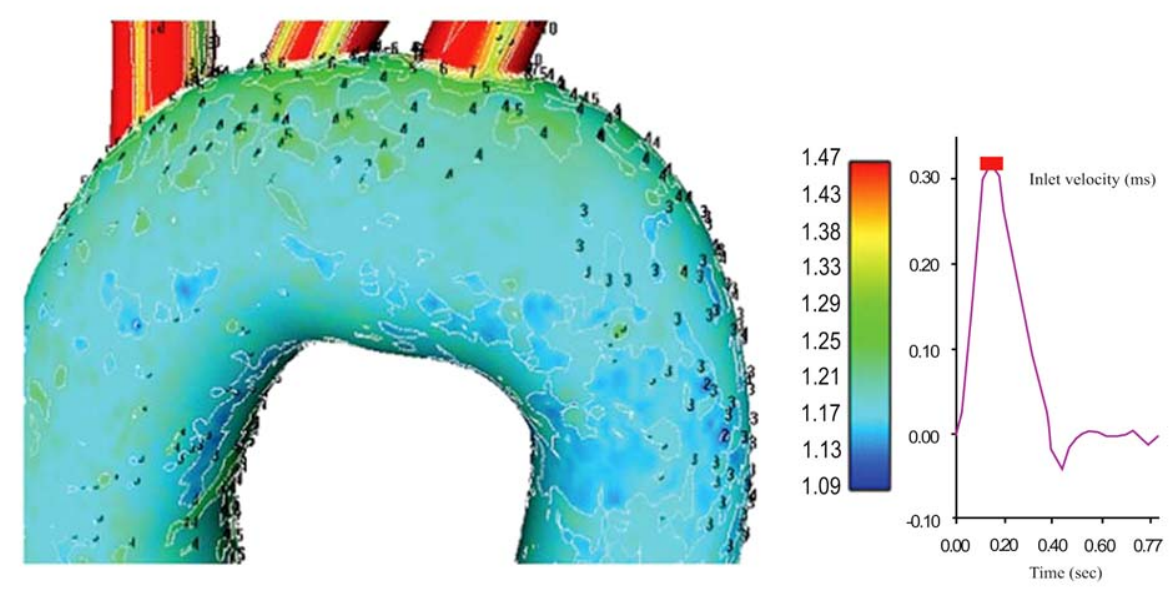

b)
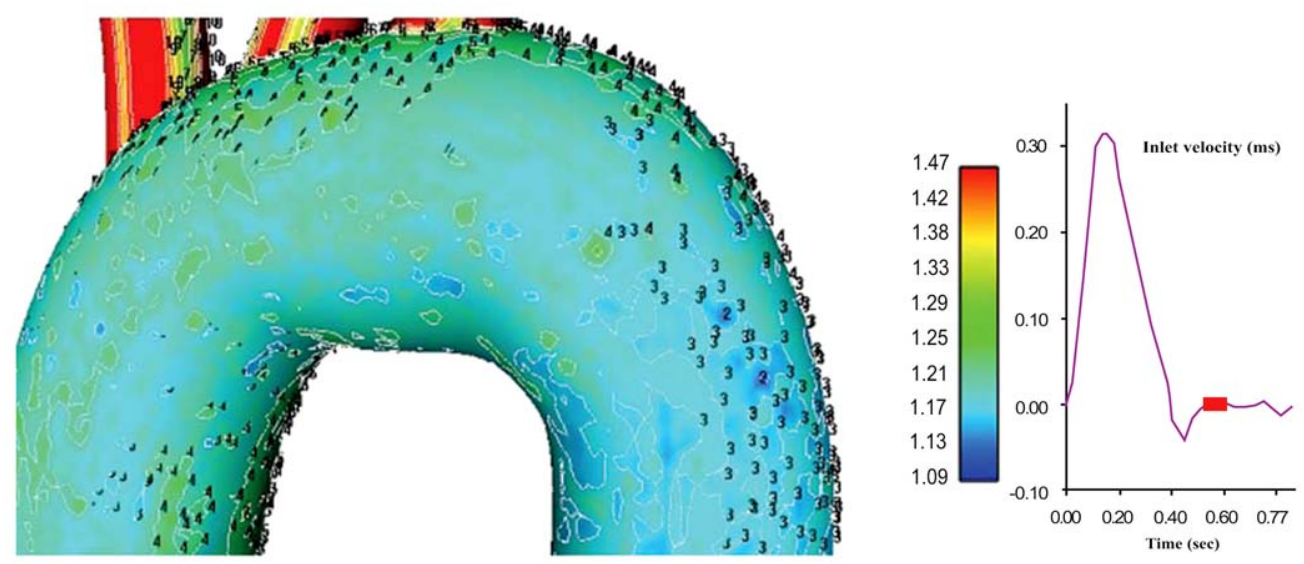

Fig. (4). Contour plots of the normalized LDL levels at the surface of the endothelium Cw/Co for the ascending-descending aorta of the aortic arch at a) peak systole and b) near minimum entrance velocity, respectively. 
a)
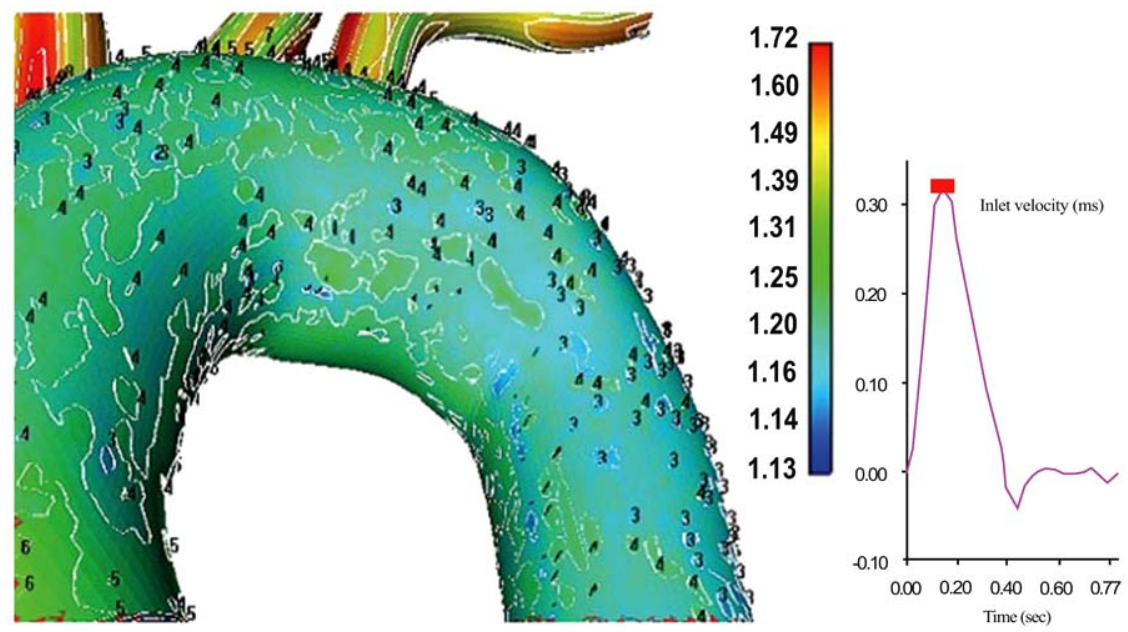

b)
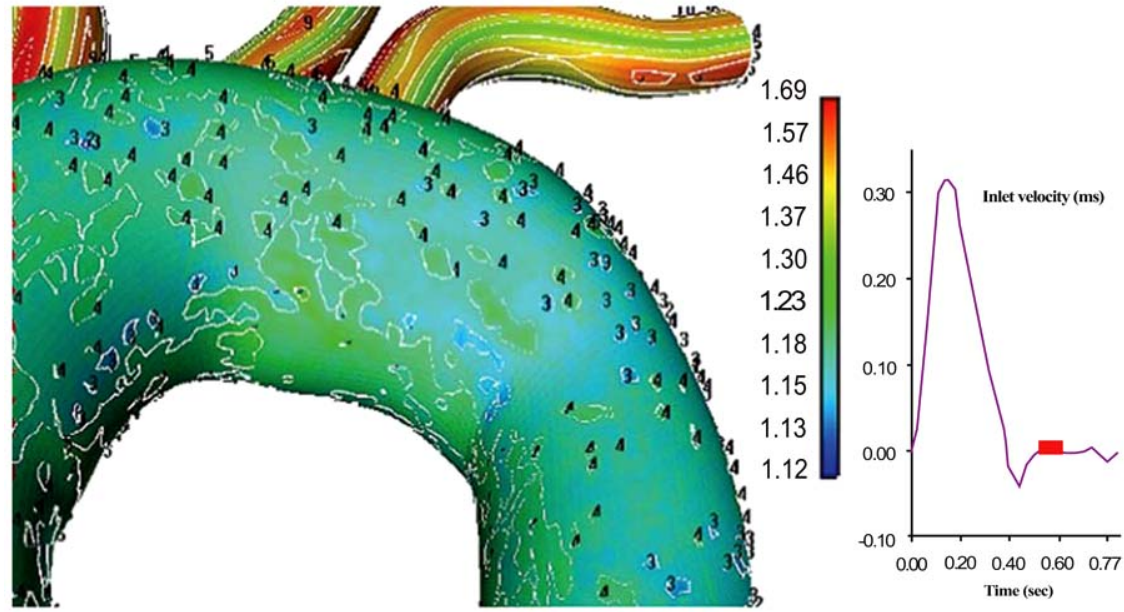

Fig. (5). Contour plots of the normalized LDL levels at the surface of the endothelium $\mathrm{Cw} / \mathrm{Co}$ for the convex side of the aortic arch at a) peak systole and $\mathbf{b}$ ) near minimum entrance velocity, respectively.

Elevated LDL regions occur at the inlet surfaces of the brachiocephalic artery, left common carotid artery as well as at the downstream regions of the left subclavian artery.

\subsection{Velocity and Strain Rate for an Ascending- Descending Aorta Transverse Section}

Velocity magnitude and strain rate distributions for an ascending-descending aorta transverse section, at peak systolic pressure and near minimum entrance velocity instant, are shown in Figs. (7 and 8), respectively.

Peak systolic pressure velocities occur in regions located close to the concave part of the early descending aorta. This is due to the centrifugal forces acting on blood flow as it encounters the aorta bend. Low blood flow velocities are encountered in the concave ascending-descending aorta at regions located just downstream to left subclavian artery. Low strain rates regions cover large area, Fig. (8), shifting towards concave parts of the ascending-descending aorta.

\subsection{Strain Rate and Molecular Viscosity at Ascending- Descending Aorta Cross-Sections}

Strain rate and molecular viscosity contour plots at various aorta cross-sections for the extreme flow instant i.e. at peak systolic and at near minimum velocity entrance, are shown in Figs. (9a, b), respectively. At peak systolic pressure, in the upstream region of the descending aorta, increased molecular viscosity and low strain rates are shifted towards the concave side and cover a relatively wide area, Fig. (9a); at the same time instant, high strain rates appear at the convex side of the upstream descending aorta region. At 
a)

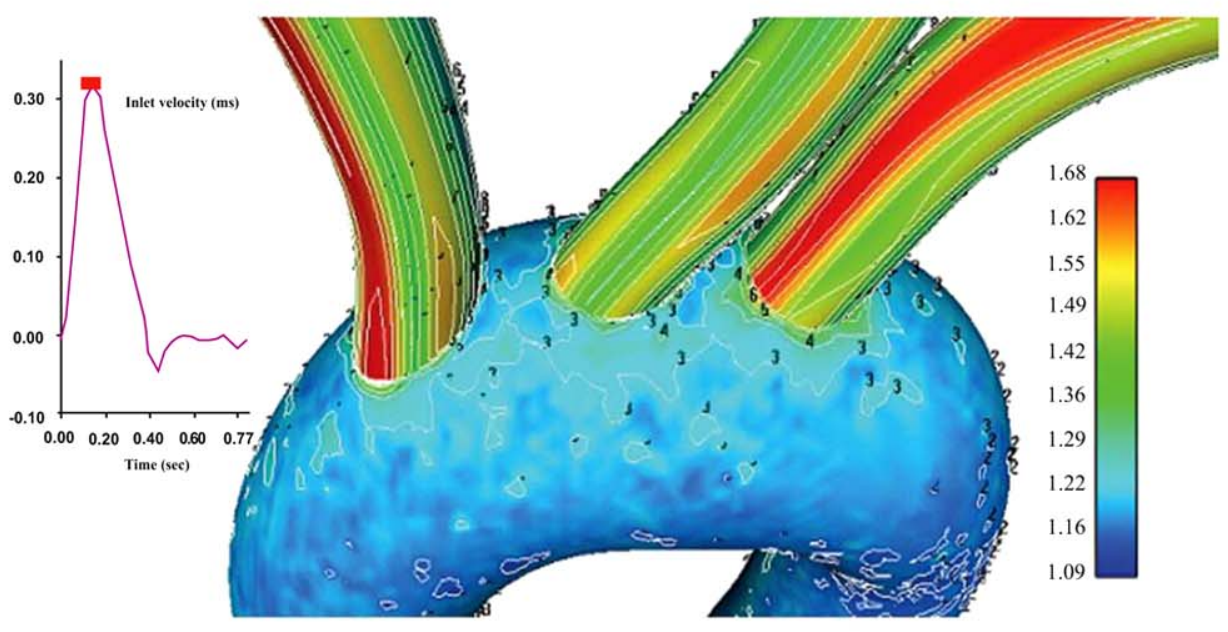

b)

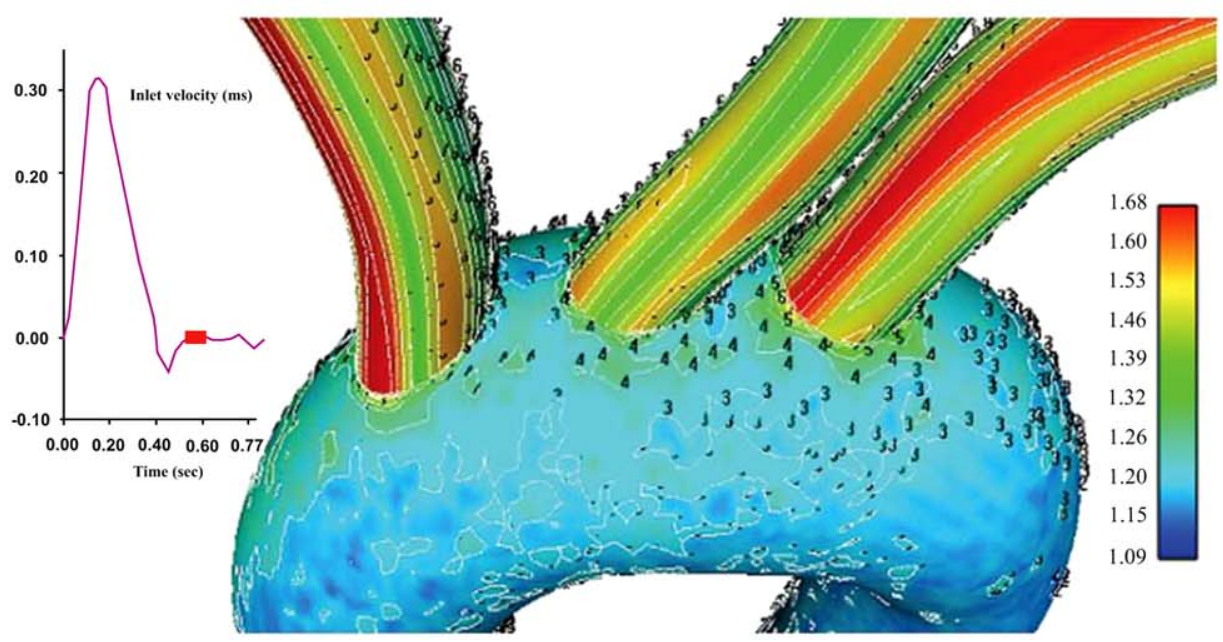

Fig. (6). Contour plots of the normalized LDL levels at the surface of the endothelium $\mathrm{Cw} / \mathrm{Co}$ for the daughter vessels of the aortic arch at a) peak systole and b) near minimum entrance velocity, respectively.

near minimum entrance velocity instant, increased strain rates are also present in the above region. Throughout the aortic arch the molecular viscosity increases as the inlet flow velocity decreases, Fig. (9b).

\subsection{LDL-WSS}

Typical relationships between LDL levels and WSS over the entire aortic arch surface at the extreme velocity instants, are shown in Figs. (10a, b). It is evident that the LDL levels at the surface of the endothelium increases with decreasing WSS. The concentration increases at a higher rate as the WSS values reduce to zero. WSS plays an important role in LDL levels at the surface of the endothelium. The dependence of LDL levels at the surface of the endothelium on
WSS is higher at near minimum entrance velocity instant, Fig. 10b, than at peak velocity instant, Fig. (10a).

\subsection{LDL Statistics}

Table 1 shows the descriptive LDL statistics for the aortic arch. The total endothelial area of the computational analyzed aortic arch is $360.69 \mathrm{~cm}^{2}$. Results, at 5 specific timepoints of the cardiac cycle, show the a) area averaged LDL levels at the surface of endothelium, b) normalized area averaged LDL levels at the surface of endothelium, c) amount of LDL taken up by all luminal aortic arch surfaces, and d) percentage LDL levels at the surface of the endothelium as compared to its lowest value taken up by all luminal aortic 

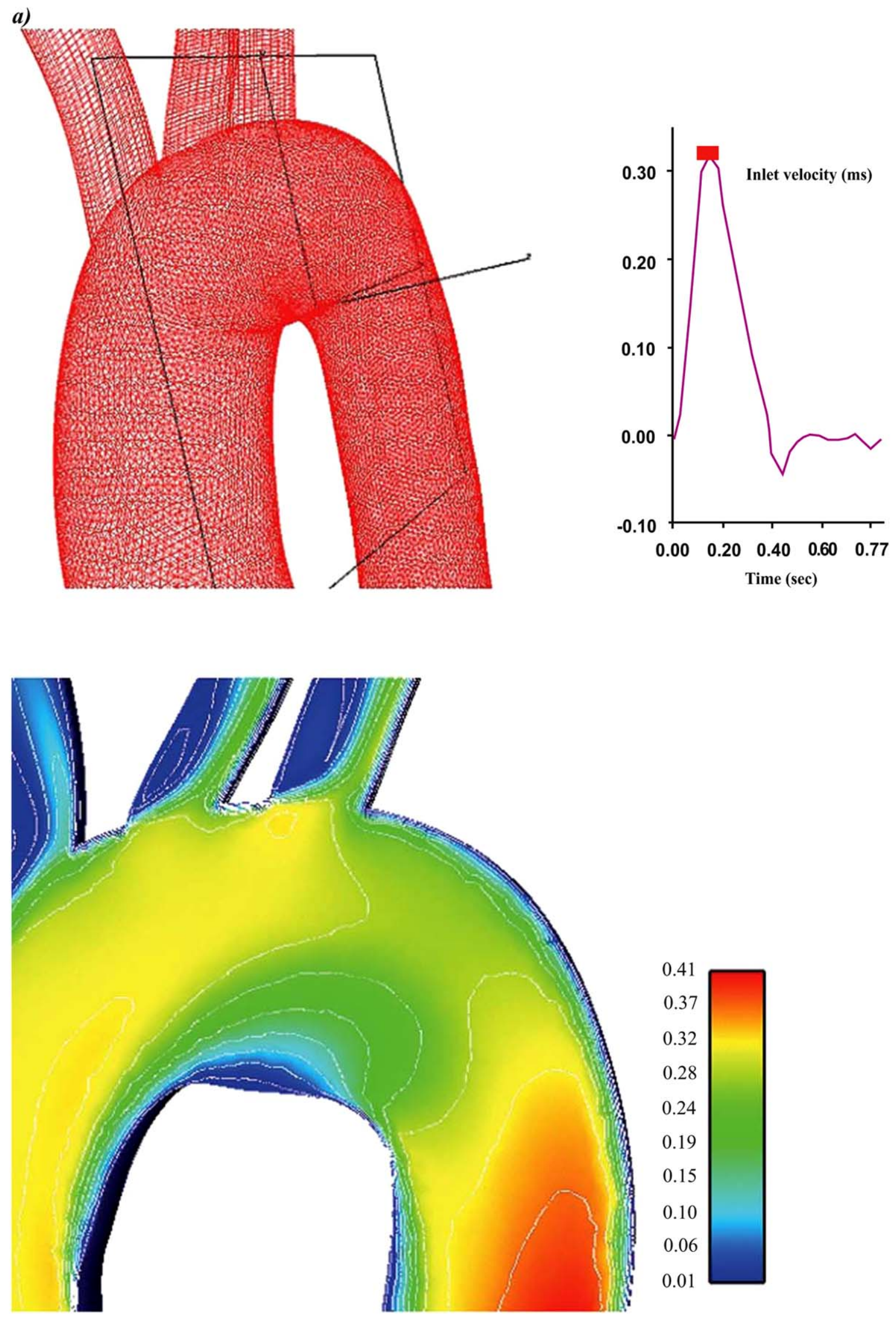
b)
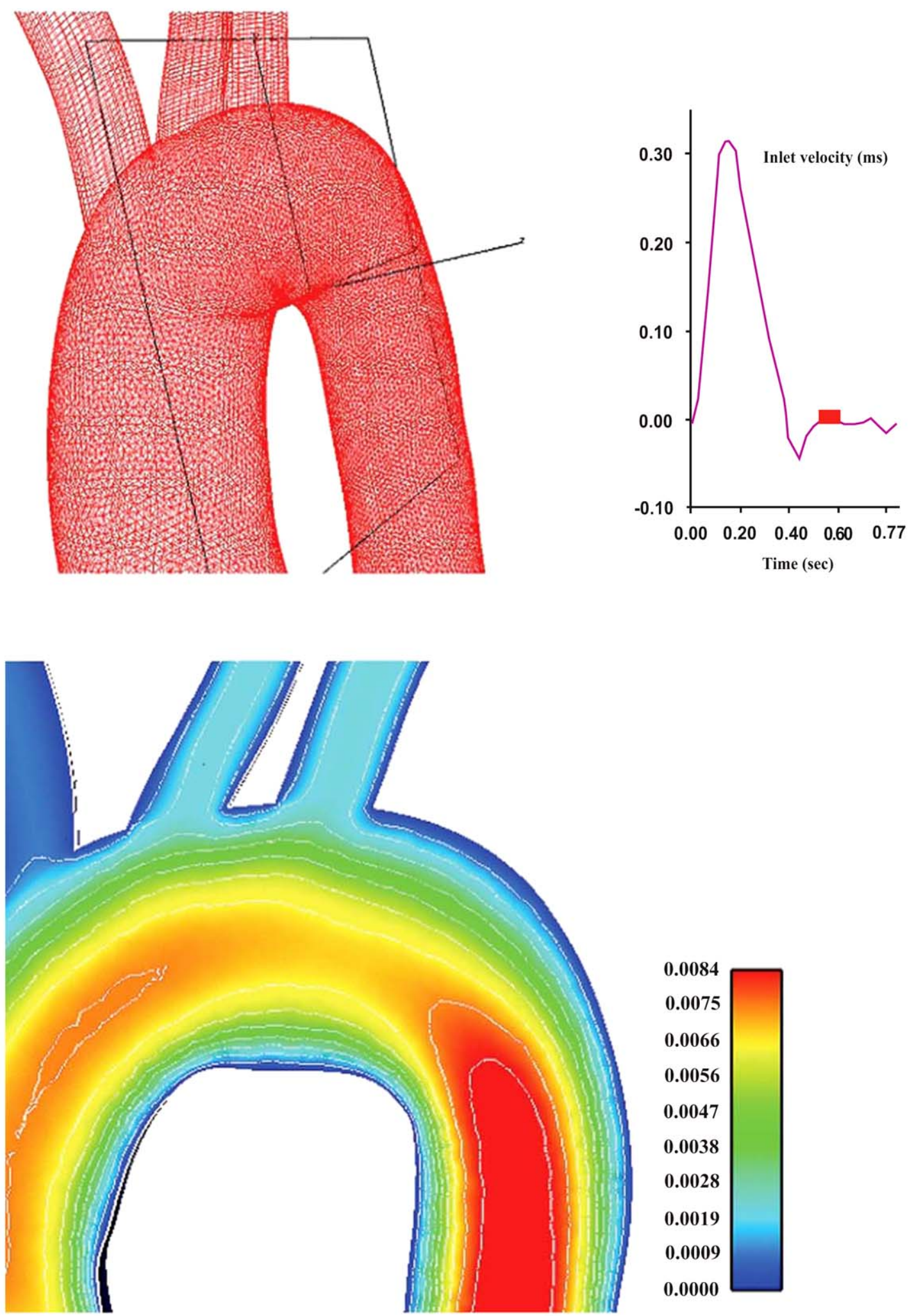

Fig. (7). Contour plots of the velocity magnitude $(\mathrm{m} / \mathrm{s})$ for an ascending-descending aorta section at a) peak systole and $\mathbf{b})$ near minimum entrance velocity, respectively. 
a)
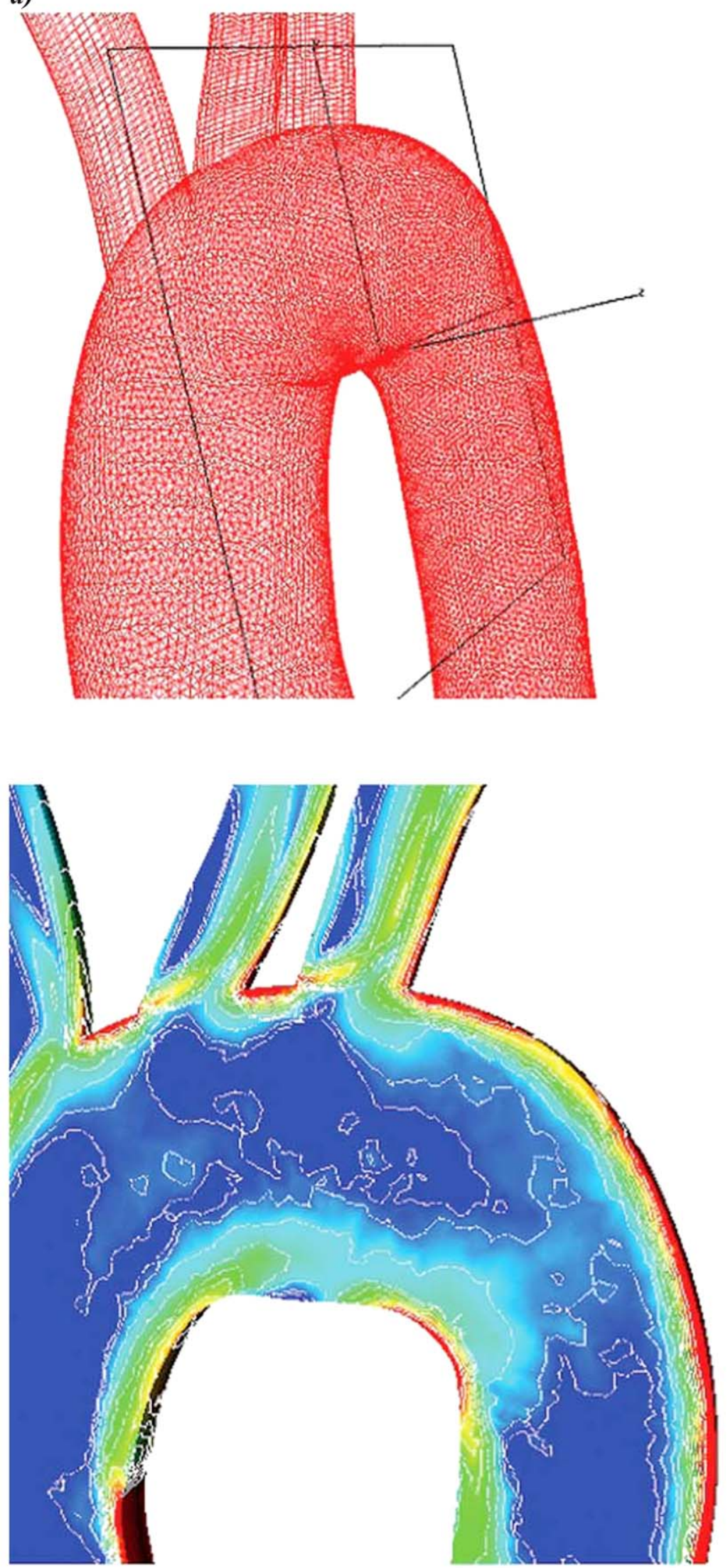

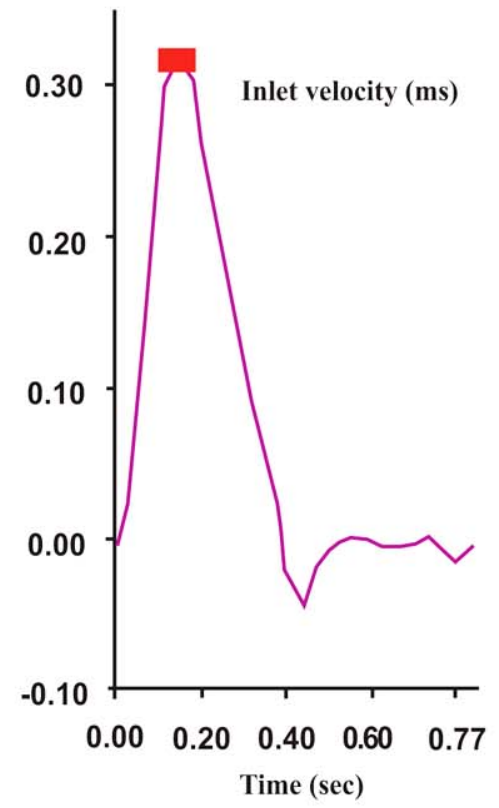

189.90
152.04
118.63
89.67
65.17
45.13
29.53
18.40
11.72
9.49 
Fig. 8. contd....

b)
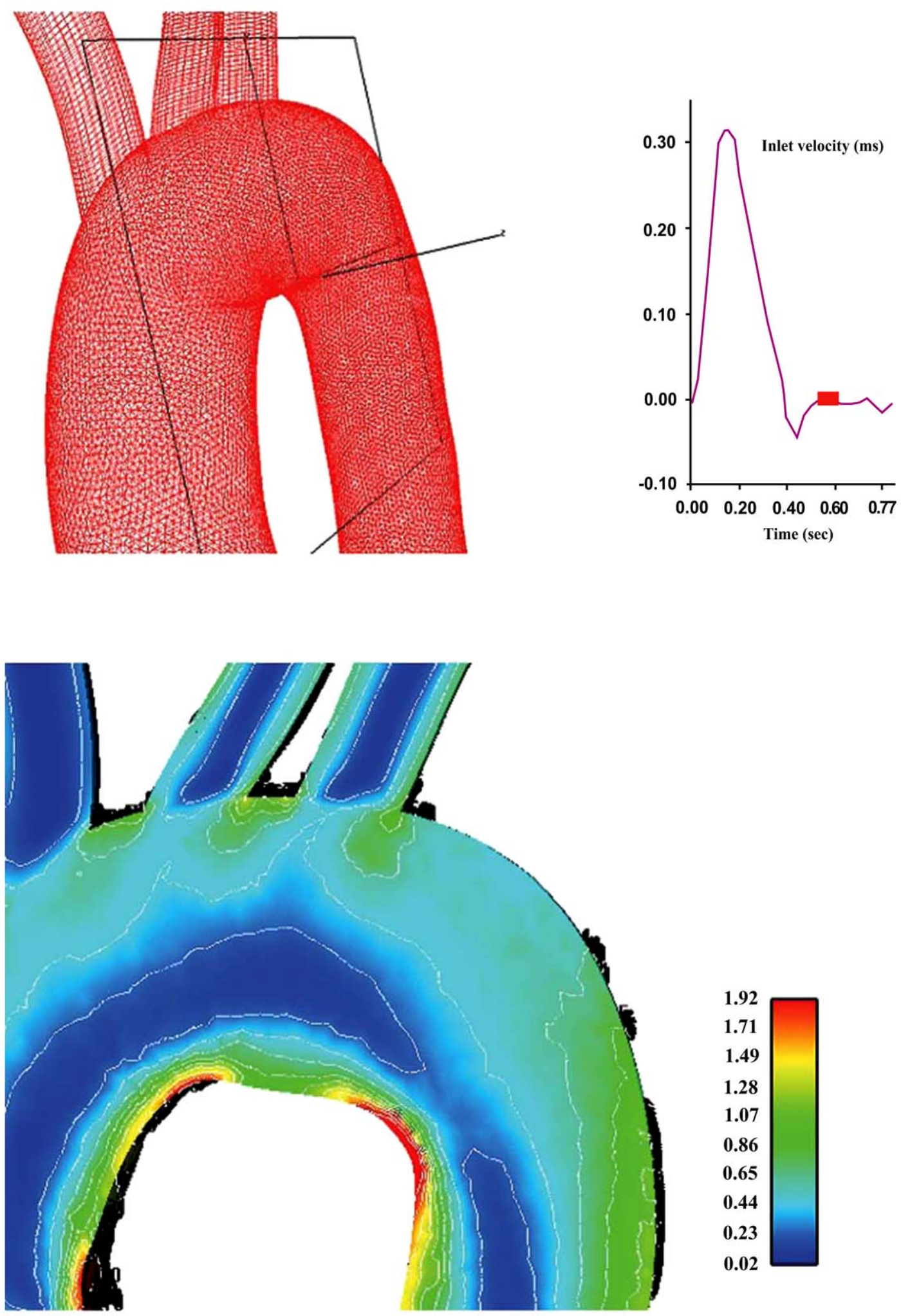

Fig. (8). Contour plots of the strain rate (1/s) for an ascending-descending aorta section at a) peak systole and b) near minimum entrance velocity, respectively. 
arch surfaces. It is worth noting that the maximum percentage increase of LDL levels at the surface of the endothelium taken up as compared with the lowest LDL at the surface of the endothelium within the cardiac cycle is $0.69 \%$.

\section{DISCUSSION}

Spatial and temporal WSS variation is important in regulating the atheroprotective, normal physiology as well as the pathobiology and dysfunction of the vessel wall through complex molecular mechanisms that promote atherogenesis. The endothelial transport properties respond instantaneously to WSS. Wherever and whenever flow becomes abnormal, the balance between the mass flow of substances from main blood stream to the arterial wall and vice versa is disrupted. This unbalanced mass transfer probably initiates arterial wall disease.

In our analysis the hydraulic conductivity of the endothelium was considered to be invariant with WSS. We computationally analyzed the LDL distribution at the surface of the endothelium and tried to elucidate the elevated LDL regions using the healthy aortic arch. Elevated LDL may be responsible for the localization and progression of atherosclerosis. The LDL substance was considered as solute within plasma. This assumption is quite helpful for computational analysis and probably it is the first step for treating the LDL as discrete particle movement. It is known that High Density Lipoprotein (HDL) facilitates the removal of the LDL from the luminal wall. However, in our work we did not consider analyzing their interaction because of the computational complexity.

The problem of mass transfer quantification is crucial in the genesis and the progression of atherosclerosis [17]. The preferred occurrence of atherosclerosis in low WSS regions is supported with extensive investigation of arterial fluid flow and mass transfer across the endothelium [20]. The action of mass transport occurs within a very thin layer, which is located very close to the wall. Any perturbation of the flow quantities, as for example high or low strain rates or

a)
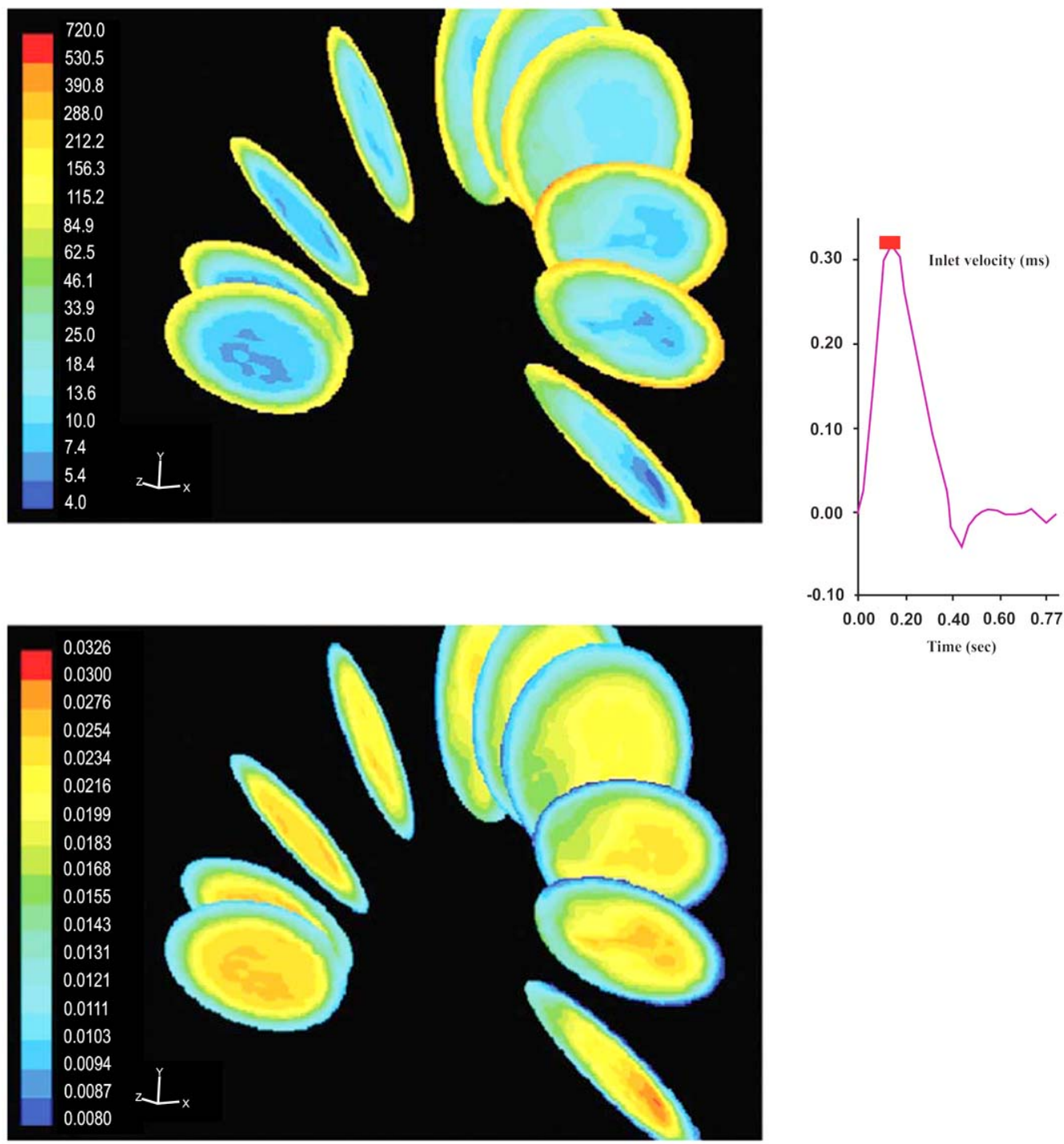
b)
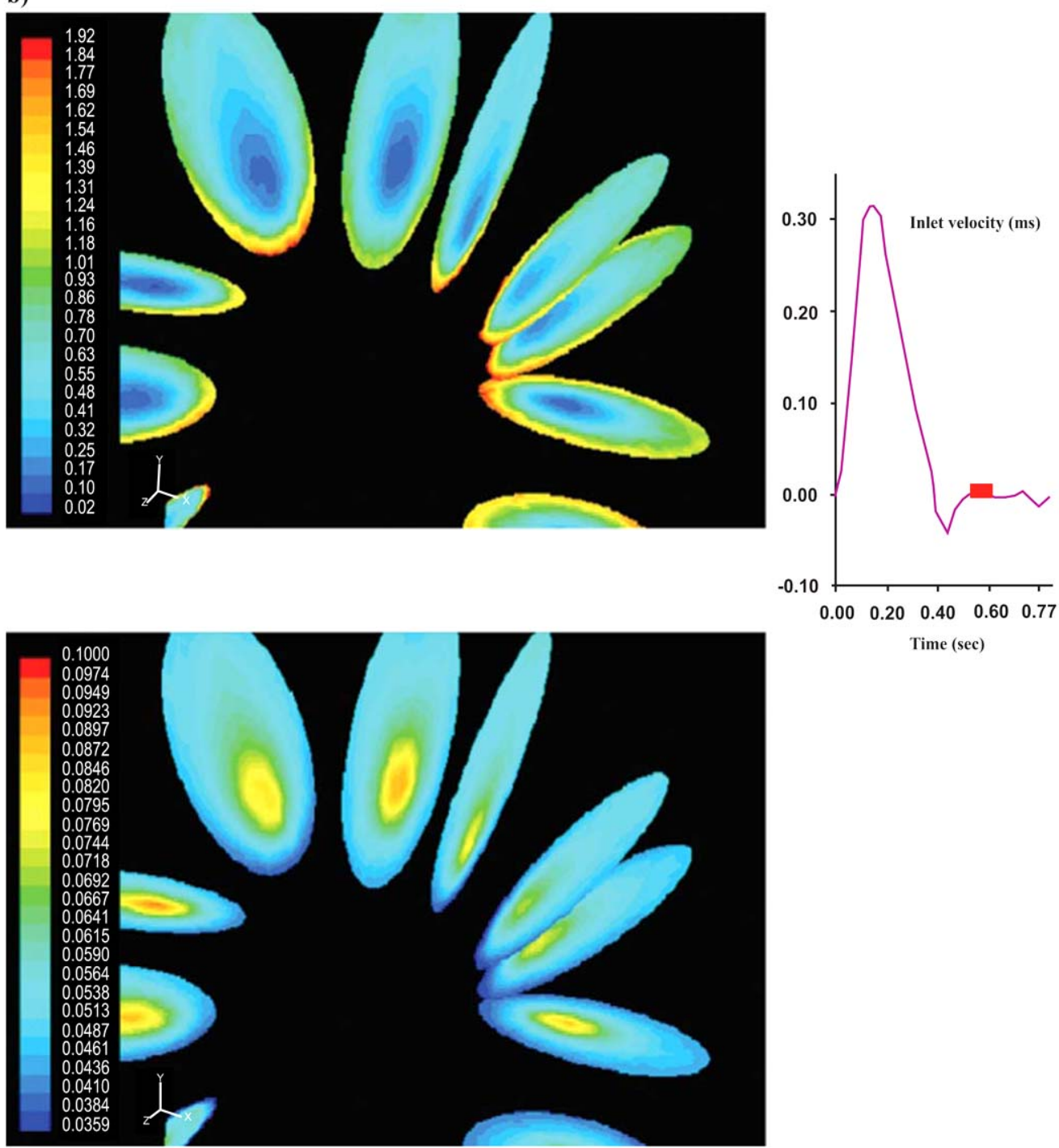

Fig. (9). Strain rate $(1 / \mathrm{s})$ and molecular viscosity $(\mathrm{kg} / \mathrm{m}-\mathrm{s})$ contour plots for ascending-descending aorta cross-sections at a) peak systole, and, b) near minimum entrance velocity, respectively.

flow reversal, in this sensitive flow region initiates mass flow disturbance to and from the arterial wall. Elongation of the endothelial cells occurs in regions of high WSS having the longest cell axis oriented parallel to the flow direction [21]. Conversely, in low WSS regions endothelial cells of polygonal shape occur and they are not oriented in any direction. It is also possible that these local alterations in endothelial cell morphology might yield different size permeability for the various blood constituents. The influences of WSS affecting the wall permeability on steady LDL transport in human right coronary arteries are starting to appear in the literature [22]. They also recognized that it is vital to consider blood pulsatility when modelling the shear-dependent macromolecular transport in large arteries.

The relationship between WSS and LDL levels indicates that the LDL is elevated at locations where WSS is low. This is a rule for all time steps. At low WSS and as it approaches zero, the LDL levels at the surface of the endothelium are rapidly increasing, Figs. (10a, b). The same relationship shows that a lot of scattered points can be found at all applied waveform time instants. This is an indication that LDL levels at the surface of the endothelium are not only WSS dependent. The LDL-WSS distribution at near minimum entrance velocity, Fig. (10b), exhibits elevated LDL levels at the surface of the endothelium at low WSS values compared with peak velocity entrance time instant, Fig. (10a). This supports the hypothesis that low WSS results into high LDL levels at the surface of the endothelium. Under the action of hemodynamics the LDL particles move to reach a particular aortic arch site. Thereafter, it is the contact time and the interaction between LDL and aortic endothelial surface which really matters. The net amount of LDL passing into arterial wall depends upon the physical properties of the wall material. High luminal surface concentration does not necessarily denote that all the LDL molecules will be transported into the arterial wall. It is the permeability of the endothelium, which largely 
a)

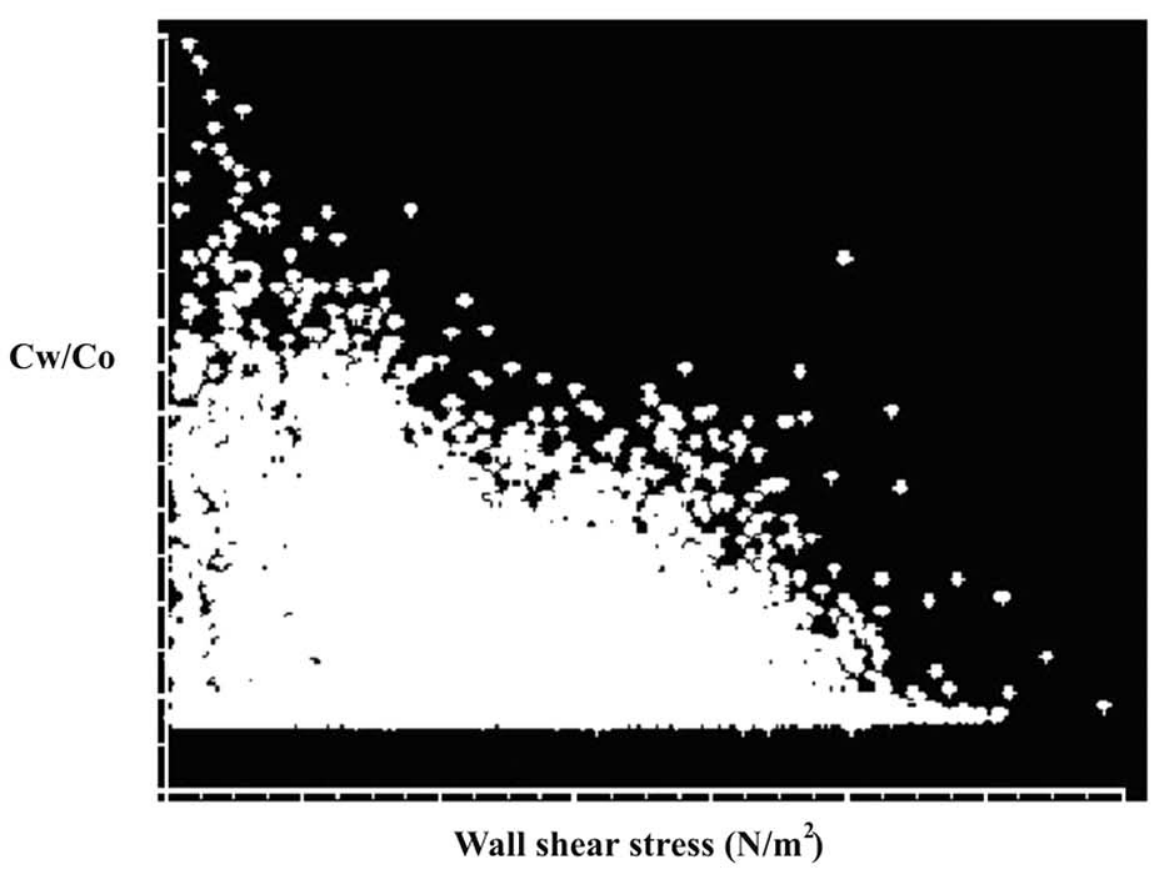

b)

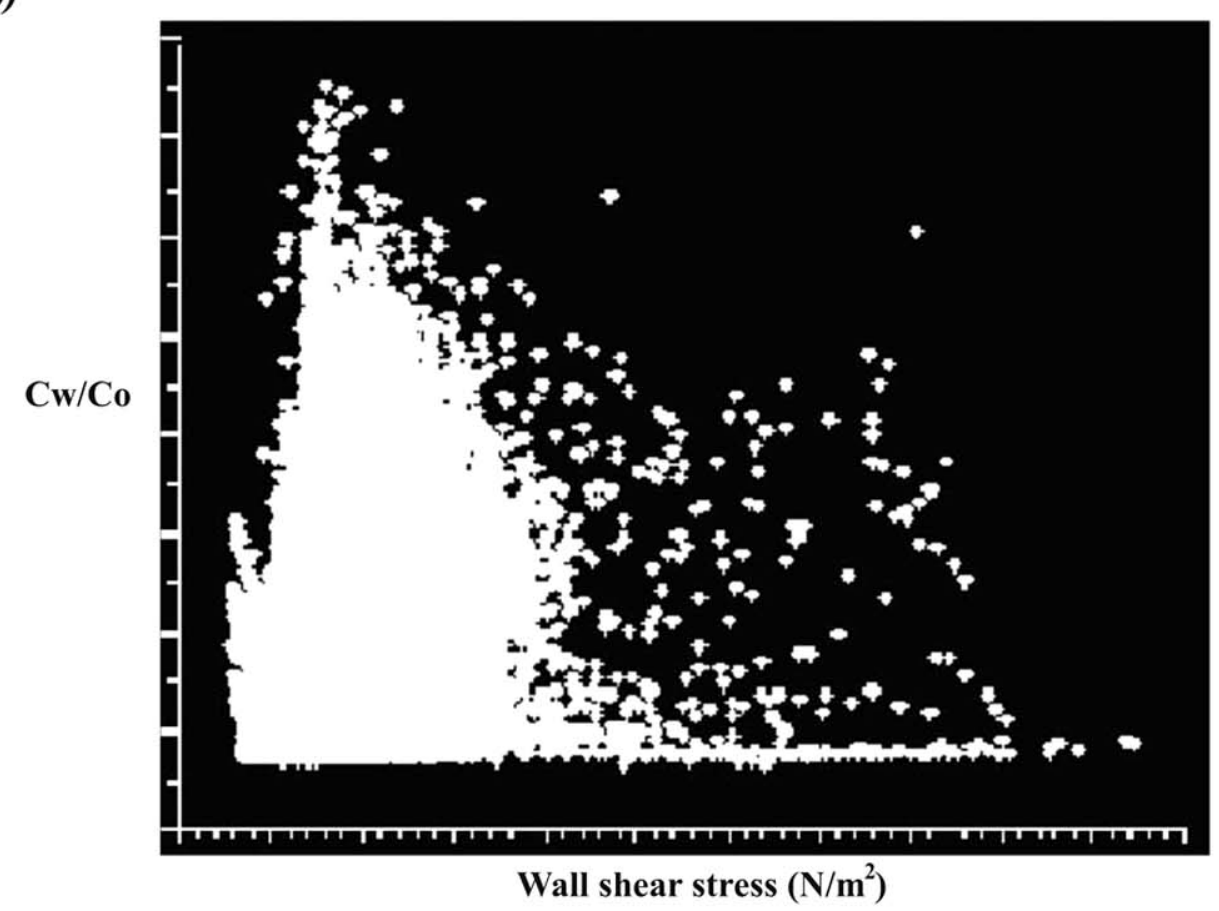

Fig. (10). Normalized LDL levels at the surface of the endothelium versus Wall Shear Stress $\left(\mathrm{N} / \mathrm{m}^{2}\right)$ for the aortic arch at a) peak systole, and, b) near minimum entrance velocity, respectively.

determines the final amount of mass passing through the wall.

Peak LDL regions of the ascending-descending aorta are located at the concave side of the descending aorta just downstream to left subclavian artery, while convex sides result in low LDL. All aortic arch walls are exposed to cholesterolemic environment although the applied mass and flow conditions refer to normal human geometry and normal mass-flow conditions.
The convex sides of the ascending-descending aortic arch exhibit, relatively to the concave side, small normalized LDL levels at all time steps. The low LDL region at peak systole, Fig. (6a), covers smaller endothelial area compared to minimum velocity entrance time instant, Fig. (6b). The mechanism of elevated LDL levels at the surface of the endothelium might also be attributed to the high curvature effects upon the flow pattern and the subsequent spatial accumulation of the circulating LDL as the mass transport 
Table 1. Descriptive Statistics of a) Luminal Surface Concentration, b) Area Averaged Normalized Luminal Surface Concentration, c) Area Averaged Amount of LDL Taken up by all Luminal Aortic Arch Surfaces, and, d) Percentage Increase of LDL as Compared with the Lowest LDL Taken up by all Luminal Aortic Arch Surfaces at Specific Time-Points (Inlet Flow Velocities) of the Cardiac Cycle

\begin{tabular}{|c|c|c|c|c|c|}
\hline $\begin{array}{c}\text { Time } \\
(\mathbf{m s e c})\end{array}$ & $\begin{array}{c}\text { Average Inlet Flow } \\
\text { Velocity }(\mathbf{m} / \mathbf{s})\end{array}$ & $\begin{array}{c}\text { a) Area Averaged LDL } \\
\text { Levels at the Surface of } \\
\text { Endothelium }(\mathbf{m g} / \mathbf{m l})\end{array}$ & $\begin{array}{c}\text { b) Area Averaged Normalized } \\
\text { LDL Levels at the Surface of } \\
\text { the Endothelium, Cw/Co }\end{array}$ & $\begin{array}{c}\text { c) LDL Taken up } \\
\text { by all Luminal } \\
\text { Surfaces (g/s) }\end{array}$ & $\begin{array}{c}\text { d) LDL Taken up as } \\
\text { Compared to the } \\
\text { Lowest LDL \% }\end{array}$ \\
\hline \hline 30.75 & 0.075 & 1.63806 & 1.27974 & $1.18167 \times 10^{-11}$ & 0.25 \\
\hline 61.50 & 0.199 & 1.63610 & 1.27820 & $1.18024 \times 10^{-11}$ & $1.17865 \times 10^{-11}$ \\
\hline 123.0 & 0.320 & 1.63389 & 1.27648 & $1.18526 \times 10^{-11}$ & 0.13 \\
\hline 286.0 & 0.026 & 1.64305 & 1.28364 & $1.18671 \times 10^{-11}$ & 0.56 \\
\hline 522.75 & 0.005 & 1.64506 & 1.28521 & 0.69 \\
\hline
\end{tabular}

equation specifies. It is the near wall paths of the velocities which might be the most important factor for elevated LDL levels at the surface of the endothelium for areas located either at the vicinity of bifurcations regions or at high curvature regions, Figs. (7, 8 and 9). These paths are strongly affected from the combined inlet velocity field (oscillation)particular geometry. Subsequently, the developing shear stress flow field and particularly the molecular viscosities dictate the WSS which in turn alters the LDL at the surface according to the physical properties of the endothelium. Because of the flow complexity and the final flow pattern in the near to endothelium regions the concave side of the ascending-descending aortic arch exhibits, relatively to the convex one, elevated LDL levels at all time steps.

Regions of low WSS values do not necessary coincide with elevated areas of LDL levels at the surface of the endothelium. At all time instants the WSS pattern clearly exhibits its low regions. The pattern of LDL, shown in Fig. (4), does not exhibit its low regions as clear as the WSS does. At near minimum entrance velocity the LDL levels at the surface of the endothelium taken up as compared with the peak systolic LDL is $0.69 \%$ higher, Table $\mathbf{1}$. It is known that atherosclerotic regions are localized into certain aortic arch areas. Atherosclerosis of the ascending aorta is a predictor of late stroke. High risk is predominantly linked to atheromas in its distal part and lesser curvature [23]. Ascending aortic and arch atheroma detected by transesophageal echocardiography is an important new independent risk factor for cerebral ischemia [24]. Furthermore, an atherosclerotic ascending aorta may be a marker of generalized atherosclerosis and thus of increased morbidity and mortality [25]. Our results show that there is elevated LDL at the surface of the endothelium in regions of low WSS. At the concave side of the descending aorta just downstream to left subclavian artery the elevated LDL sites coincide with Farthing findings [26]. How these findings influence local pathology (e.g. aortic dissection, aneurysm formation or aortic arch emboli) remains to be elucidated.

The assumption, that wall permeability to LDL is constant throughout needs to be altered. A novel aspect will be that the permeability is a function of either the WSS or spatial gradient of WSS. Arterial wall deformation needs to be taken into consideration, since the flow pattern-mass transport is seriously determined from the geometrical configuration of the artery. Arterial movement has to be taken into account since the applied acceleration force will yield a different flow field. Hypolipidaemic and antihypertensive agents may affect the localization of aortic arch atherosclerotic regions; this possibility should be investigated. Finally, the percentage of LDL deposition at the surface of the endothelium, in terms of the circulating LDL quantity, needs to be analyzed.

\section{CONCLUSIONS}

This computational study elucidates the LDL transport in relation to the WSS in the normal human aortic arch under oscillating flow conditions at rest. LDL levels at the surface of the arterial wall endothelium are flow-dependent, exhibiting increased permeation of LDL at specific regions. Concave sides of the aortic arch exhibit, relatively to the convex ones, elevated LDL levels at the surface of the endothelium at all times. Aortic arch walls are exposed to cholesterolemic environment although the applied mass and flow conditions refer to normal human geometry and normal mass-flow conditions. Low WSS regions are exposed to high LDL levels at the surface of the endothelium. Regions of high LDL levels at the surface of the endothelium do not necessarily co-locate to the sites of low WSS. For the peak entrance velocity, in the ascending-descending aorta region, the LDL level at the surface of the endothelium is $23.0 \%$ higher than that at entrance. The corresponding LDL levels at the surface of the endothelium for the near minimum entrance velocity reaches $26.0 \%$. The net amount of LDL mass per second taken up by all luminal aortic arch surfaces, also at peak velocity, is $1.17865 \times 10^{-11} \mathrm{~g} / \mathrm{s}$. The highest area averaged normalized LDL taken up as compared with the lowest one is $0.69 \%$. The near wall paths of the velocities might be the most important factor for elevated LDL levels at the surface of the endothelium.

\section{REFERENCES}

[1] Soulis JV, Farmakis TM, Giannoglou GD, Louridas GE. Wall shear stress in normal left coronary artery tree. J Biomech 2006; 39: 742-79.

[2] Ethier CR. Computational modeling of mass transfer and links to atherosclerosis. Ann Biomed Eng 2002; 30: 461-71. 
[3] Friedman MH, Deters OJ, Mark FF, Bargeron CB, Hutchins GM. Arterial geometry affects hemodynamics. A potential risk factor for atherosclerosis. Atherosclerosis 1983; 46: 225-31.

[4] Giannoglou GD, Soulis JV, Farmakis TM, Farmakis DM, Louridas GE. Hemodynamic factors and the important role of local low static pressure in coronary wall thickening. Int J Cardiol 2002; 86: $27-40$.

[5] Soulis JV, Farmakis TM, Giannoglou GD, et al. Molecular viscosity in the normal left coronary arterial tree. Is it related to atherosclerosis? Angiology 2006; 57: 33-40.

[6] Soulis JV, Farmaki TM, Giannoglou GD, Louridas GE, Parcharidis GE. Spatial and phasic oscillation of non-Newtonian wall shear stress in human left coronary artery bifurcation, an insight to atherogenesis. Coron Artery Dis 2006; 17: 351-8.

[7] Ogunrinade O, Kameya GT, Trusky GA. Effect of fluid shear stress on the permeability of the arterial endothelium. Ann Biomed Eng 2002; 30: 430-46. Review.

[8] Fatouraee N, Deng X, De Champlain A, Guidoin R. Concentration polarization of low density lipoproteins (LDL) in the arterial system. Ann N Y Acad Sci 1998; 11: 137-46.

[9] Wada S, Karino T. Theoretical prediction of low-density lipoprotein concentration at the luminal surface of an artery with a multiple bend. Ann Biomed Eng Bioemd 2002; 30: 778-91.

[10] Stangeby DK, Ethier CR. Computational analysis of coupled blood-wall arterial LDL transport. J Biomech Eng 2002; 124: 1-8.

[11] Karino T. Theoretical prediction of low-density lipoproteins concentration at the luminal surface of an artery with a multiple bend. Ann Biomed Eng 2002; 30: 778-90.

[12] Sun N, Wood N, Hughes S, Thom S, Xu X. Influence of pulsatile flow on LDL transport in the arterial wall. Ann Biomed Eng 2007; 35: 1782-90.

[13] Prosi M, Zunino P, Perktold K, Quarteroni A. Mathematical and numerical models for transfer of low-density lipoproteins through the arterial walls: a new methodology for the model set up with applications to the study of disturbed luminal flow. J Biomech 2005; 8: 903-17.

[14] Wei L, Wen GB, Tan WC. Blood flow and macromolecular transport in curved blood vessels. Appl Math Mech (English Edition), 2006; 27: 1223-31.
[15] Deng X, Marois Y, How T, Mehri Y, King M, Guidoin R. Luminal surface concentration pf lipoprotein (LDL) and its effect on the wall uptake of cholesterol by canine carotid arteries. J Vasc Surg 1995; 21: 135-45.

[16] Yang N, Vafai K. Modeling of low-density lipoprotein (LDL) transport in the artery-effects of hypertension. Int J Heat Mass Transfer 2006; 49: 850-67.

[17] Lei M, Kleinstreuer C, Truskey G. A focal stress gradientdependent mass transfer mechanism for atherogenesis in branching arteries. Med Eng Phys 1996; 4: 326-32.

[18] Naiki T, Sugiyama H, Tashiro R, Karin T. Flow-dependent concentration polarization of plasma proteins at the luminal surface of a cultured endothelial cell monolayer. Biorheology 1999; 36: 22541.

[19] Murray CD. The physiological principle of minimum work. I. The vascular system and the cost of blood volume. Proc Natl Acad Sci USA 1926; 12: 207-14.

[20] Caro CG. Discovery of the role of wall shear in atherosclerosis. Arterioscler Thromb Vasc Biol 2008.

[21] Davies PF, Tripathi SC. Mechanical stress mechanisms and the cell. An endothelial paradigm. Circ Res 1993; 72: 239-45.

[22] Sun N, Torii R, Wood NB, Hughes AD, Thom SA, Xu XY. Computational modeling of LDL and albumin transport in an in vivo CT image-based human right coronary artery. J Biomech Eng 2009; 131: 021003.

[23] Van der Linden J, Bergman P, Hadjinikolaou L. The topography of aortic atherosclerosis enhances its precision as a predictor of stroke. Ann Thorac Surg 2007; 83(6): 2087-92.

[24] Jones EF, Kalman JM, Calafiore P, Tonkin AM, Donnan GA Proximal aortic atheroma: An independent risk factor for cerebral ischemia. Stroke 1995; 26: 218-24.

[25] Davila-Roman VG, Murphy SF, Nickerson NJ, Schechtman KB, Barzilai B. Atherosclerosis of the ascending aorta is an independent predictor of long-term neurologic events and mortality. J Am Coll Cardiol 1999; 33:1308-16.

[26] Farthing S. Flow in the thoracic aorta and its relation to atherogenesis. PhD Thesis, University of Cambridge 1978.

This is an open access article licensed under the terms of the Creative Commons Attribution Non-Commercial License (http://creativecommons.org/licenses/by$\mathrm{nc} / 3.0 /$ ) which permits unrestricted, non-commercial use, distribution and reproduction in any medium, provided the work is properly cited. 